\title{
ESTUDIO PRELIMINAR DE LA CONTRACCIÓN Y EL COLAPSO EN EUCALYPTUS NITENS
}

\author{
PRELIMINARY STUDY OF SHRINKAGE AND COLLAPSE \\ IN EUCALYPTUS NITENS
}

Rubén A. Ananías ${ }^{1}$, Cinthya Díaz, Laura Leandro ${ }^{2}$

\section{RESUMEN}

En este trabajo se estudió la contracción y el colapso en familias de Eucalyptus nitens.

La madera fue recogida de 15 árboles en pie de 17 años de edad creciendo en plantaciones de la Octava Región de Chile. Fueron seleccionados al azar tres árboles provenientes de cinco familias de $E$. nitens. Las probetas para los ensayos de contracción y colapso fueron preparadas a partir de una muestra obtenida de una rodela extraida a la altura del DAP de cada árbol. Los ensayos de contracción y colapso fueron realizados de acuerdo a las normas chilenas.

Fueron determinados la contracción, el colapso, el coeficiente de contracción unitario y el punto de intersección de la contracción. Los resultados evidenciaron que la contracción y el colapso aumentaron con la posición radial y una alta contracción e intenso colapso en la zona central y lateral fueron asociados a una baja de la densidad en estas zonas.

Palabras clave: Contracción transversal, colapso, eucalipto, Chile.

\begin{abstract}
In this study the variation of transversal shrinkage and collapse in Eucalyptus nitens were evaluated.

Fifteen trees, three of each five different families were collected from plantations of E. nitens 17 years-old, growing in the Eighth Region of Chile. The wood samples of shrinkage and collapse was obtained from the first log, in three positions from the pith to bark south (core, transition and lateral). Wood samples were prepared and then acclimatized in according to the Chilean Standards. The collapse was determined by the difference of shrinkage before and after reconditioning. Shrinkage unit coefficients and the shrinkage intersection points were also determined.
\end{abstract}

The results showed variability radial transversal shrinkage and collapse of between trees and families of $E$. nitens. The transversal shrinkage and collapse tended to increase with radial position, despite that higher transversal shrinkage and collapse were more intense in the core or transition zones due to lower densities.

Keywords: Transversal shrinkage, collapse, Eucalyptus nitens, Chile.

\footnotetext{
${ }^{1}$ Departamento de Ingeniería en Maderas, Facultad de Ingeniería, Universidad del Bío-Bío.Concepción. Chile

${ }^{2}$ Estudiante Doctorado en Ciencias e Industrias de la Madera, Departamento de Ingeniería en Maderas, Facultad de Ingeniería,

Universidad del Bío-Bío. Concepción. Chile

Autor para correspondencia: ananias@ubiobio.cl

Recibido: 25.05.2009. Aceptado: 05.11.2009.
} 


\section{INTRODUCCION}

El Eucalyptus nitens, fue plantado originalmente en Chile, para abastecer de materia prima a la industria de la celulosa y papel, no obstante ha estado siendo evaluado para su utilización en productos sólidos de mayor valor agregado, madera aserrada, muebles, chapas, tableros (INFOR 1989, 2004). Su utilización como madera sólida de mayor valor agregado es limitada por un alto porcentaje de grietas internas y superficiales, alta contracción e intenso colapso (Haslett 1988, Sepúlveda y Urrutia 1996, Galleti 1996, Leandro et al. 2008a, Washusen and Innes 2008, Washusen et al. 2008)

Respecto de la contracción se ha observado una alta anisotropía de la contracción transversal. De acuerdo con Sepúlveda y Urrutia (1996) la anisotropía de la contracción transversal en E. nitens es de hasta 3. Mientras que Galleti (1996) encuentra que la contracción total del E. nitens durante el secado hasta $10 \%$ de contenido de humedad es de $5.5 \%$ y $14,1 \%$ en dirección radial y tangencial respectivamente. En relación al colapso, Rozas et al. (2005) en ensayos de secado a escala industrial observaron una alta contracción volumétrica total debido al colapso de esta madera. En adición, Hamilton et al. 2009 muestran que la contracción y el colapso pueden ser interrelacionados para mejorar la selección genética en E. nitens, para productos de mayor valor agregado.

En este contexto el presente estudio tiene como objetivo determinar la contracción y el colapso en árboles provenientes de cinco familias de Eucalyptus nitens.

\section{METODOLOGIA EXPERIMENTAL}

\section{Selección de árboles}

Los árboles de E. nitens (procedencia Toorongo, Australia) de 16 años fueron seleccionados de bosques plantados el año 1990 en un ensayo de progenie en un sitio tipo trumao, en la Hacienda Rucamanqui (Yungay, Provincia de Ñuble, Octava Región de Chile). Se seleccionaron 15 árboles, correspondientes a 5 familias de 3 árboles cada una, que se detallan en Tabla 1. Los árboles fueron marcados convenientemente respecto de la posición sur-norte y después del volteo se marcaron las trozas con las 4 posiciones cardinales. Las trozas fueron mantenidas frigorizadas $\left(-10^{\circ} \mathrm{C}\right)$ y luego bajo agua en una piscina en el laboratorio de secado de la Universidad del Bío Bío para mantenerlas en condición saturada hasta el momento de ser utilizadas.

Tabla 1: Características de árboles con los que se realizaron los ensayos (Fuente: Leandro et al. 2007)

\begin{tabular}{|c|c|c|c|c|}
\hline FAMILIA & ÁRBOL & ALTURA & DIÁMETRO (cm) & DENSIDAD BÁSICA (kg/m $\left.\mathbf{m}^{\mathbf{3}}\right)$ \\
\hline \multirow{3}{*}{1} & 1 & DAP & 36.0 & 510 \\
\cline { 2 - 5 } & 2 & DAP & 67.6 & 536 \\
\cline { 2 - 5 } & 3 & DAP & 46.6 & 494 \\
\hline \multirow{3}{*}{2} & 4 & DAP & 59.3 & 452 \\
\cline { 2 - 5 } & 5 & DAP & 46.0 & 436 \\
\cline { 2 - 5 } & 6 & DAP & 49.8 & 436 \\
\hline \multirow{3}{*}{3} & 7 & DAP & 44.9 & 471 \\
\cline { 2 - 5 } & 8 & DAP & 51.4 & 447 \\
\hline \multirow{3}{*}{4} & 9 & DAP & 42.2 & 515 \\
\cline { 2 - 5 } & 10 & DAP & 43.7 & 452 \\
\cline { 2 - 5 } & 11 & DAP & 41.1 & 499 \\
\hline \multirow{3}{*}{5} & 12 & DAP & 51.6 & 499 \\
\cline { 2 - 5 } & 14 & DAP & 37.4 & 452 \\
\cline { 2 - 5 } & 15 & DAP & 47.2 & 466 \\
\hline
\end{tabular}




\section{Preparación de la madera}

Para este estudio se ocupó la troza proveniente de la base de cada árbol (al DAP). Las trozas fueron transportadas al Pabellón Tecnológico de la Madera (PTM) de la UBB, donde fueron partidas a la mitad en un carro huincha, luego se dieron vuelta para que la misma máquina cortara los costados para obtener el listón de la posición sur. Luego el listón se dividió en tres secciones: central C, transición T y lateral L. De este listón se obtuvieron probetas normalizadas de 25 x 25 x $100 \mathrm{~mm}$ en corte $100 \%$ radial, según la Norma Chilena Oficial 176/3 (INN, 1986). Las probetas fueron obtenidas en las 3 posiciones desde médula a corteza, $(\mathrm{C}, \mathrm{T}$ y $\mathrm{L})$, teniendo cuidado que siempre coincidieran en el mismo numero de anillos. De cada una de estas secciones se prepararon cuatro probetas de cada posición.

Una vez cortadas y cepilladas las probetas fueron inmediatamente identificadas con códigos para reconocer su procedencia. Además fueron marcadas en tres puntos equidistantes en sus caras transversales y una en el largo con el fin de medir sus dimensiones siempre en los mismos puntos.

En total se recogieron 149 probetas de acuerdo al diseño experimental de la Tabla 2.

Tabla 2: Diseño experimental

\begin{tabular}{|c|c|c|c|c|}
\hline FAMILIA & ÁRBOL & $\begin{array}{l}\text { PROBETAS } \\
\text { POR ÁRBOL }\end{array}$ & $\begin{array}{l}\text { PROBETAS } \\
\text { POR FAMILIA }\end{array}$ & TOTAL \\
\hline \multirow{3}{*}{1} & 1 & 8 & \multirow{3}{*}{28} & \multirow{15}{*}{149} \\
\hline & 2 & 12 & & \\
\hline & 3 & 8 & & \\
\hline \multirow{3}{*}{2} & 4 & 8 & \multirow{3}{*}{24} & \\
\hline & 5 & 11 & & \\
\hline & 6 & 5 & & \\
\hline \multirow{3}{*}{3} & 7 & 12 & \multirow{3}{*}{34} & \\
\hline & 8 & 11 & & \\
\hline & 9 & 11 & & \\
\hline \multirow{3}{*}{4} & 10 & 12 & \multirow{3}{*}{31} & \\
\hline & 11 & 12 & & \\
\hline & 12 & 7 & & \\
\hline \multirow{3}{*}{5} & 13 & 12 & \multirow{3}{*}{32} & \\
\hline & 14 & 12 & & \\
\hline & 15 & 8 & & \\
\hline
\end{tabular}

\section{Acondicionado de las probetas}

Una vez preparadas, identificadas y marcadas las 149 probetas fueron mantenidas en agua hasta iniciar los ensayos experimentales. Todas las probetas fueron medidas y pesadas inicialmente y luego acondicionadas a $25 \pm 2^{\circ} \mathrm{C}$ y $65 \pm 5 \%$ de humedad relativa de acuerdo a la norma chilena $176 / 3$ (INN, 1984). Para ello fue utilizada una cámara Heraeus Votsch, ubicada en los laboratorios de secado de la Universidad del Bío-Bío. Los datos de temperatura de bulbo seco y bulbo húmedo en el interior de la cámara de clima, fueron recogidos continuamente cada 15 minutos mediante un sistema de adquisición de datos (Fluke data-logger). 


\section{Mediciones experimentales}

Periódicamente fueron pesadas y medidas todas las probetas usando un vernier digital (Mitutoyo $0.1 \mathrm{~mm}$ ) y una balanza de precisión (Wagner $0.01 \mathrm{~g}$ ). Una vez que las probetas alcanzaron peso constante en la cámara de clima, se procedió a reacondicionarlas en vapor saturado a $100{ }^{\circ} \mathrm{C}$ por 3 horas, en un recipiente termostático. Luego las probetas fueron acondicionadas nuevamente en la cámara de clima, hasta peso constante con el fin de alcanzar el mismo contenido de humedad anterior al reacondicionado.

\section{Determinación de contracción y colapso}

La contracción (C) y el colapso (Co) fueron determinados en base a la norma chilena oficial 176/3 (INN 1984). Para la contracción se utilizó la expresión siguiente

$$
C=\frac{D_{\text {inicial }}-D_{\text {actual }}}{D_{\text {inicial }}} \cdot 100
$$

donde:

$\mathrm{D}_{\text {inicial }}=$ dimensión de la probeta, en milímetros, al contenido de humedad máximo.

$\mathrm{D}_{\text {actual }}=$ dimensión de la probeta, en milímetros, al contenido de humedad que se indique

La contracción volumétrica $(\mathrm{Cv})$ fue determinada como la sumatoria de las contración radial $(\mathrm{Cr}) \mathrm{y}$ tangencial $(\mathrm{Ct})$

$$
C v=C r+C t
$$

El colapso (Co) fue determinado por la diferencia de contracción antes del reacondicionado (Car) y después del reacondicionado (Cdr)

$$
C o=C a r-C d r
$$

\section{Calculo del coeficiente de contracción unitario $(\mathrm{Cu})$ y el punto de intersección de la contracción} (PIC)

El Cu y el PIC fueron determinados después del reacondicionado. El Cu se calculó por la relación siguiente

$$
C u=\frac{C_{T 0}-C_{T d r}}{C H_{d r}}
$$


donde:

$\mathrm{C}_{\mathrm{T} 0}=$ contracción transversal (radial o tangencial) de la madera en estado anhidro.

$\mathrm{C}_{\mathrm{Tdr}}=$ contracción transversal (radial o tangencial) de la madera, después del reacondicionado.

$\mathrm{CH}_{\mathrm{dr}}=$ contenido de humedad de la madera, después del reacondicionado.

Y el PIC se determinó prolongando la porción recta de la curva de contracción hasta interceptar al eje de las abscisas ( $0 \%$ de contracción), esto es,

$$
P I C=\frac{C_{T N \max }}{C u}
$$

donde:

$\mathrm{C}_{\mathrm{TNmax}}=$ contracción transversal (radial o tangencial) de la madera normal máxima.

\section{Determinación de densidad básica $\left(\mathrm{S}_{\mathrm{o}, \mathrm{g}}\right)$ y contenido de humedad máximo $\left(\mathrm{CH}_{\mathrm{g}}\right)$}

La densidad básica $\left(\mathrm{S}_{0 \mathrm{~g}}\right)$, se determinó de acuerdo a la norma chilena NCh 176/2 of. 86 (INN 1988), al igual que el contenido de humedad máximo $\left(\mathrm{CH}_{\mathrm{g}}\right)$. El volumen máximo $\left(\mathrm{V}_{\mathrm{g}}\right)$ en $\mathrm{m}^{3}$ fue determinado en base a las mediciones de las dimensiones de las probetas y con la masa anhidra $\left(\mathrm{M}_{0}\right)$ en $\mathrm{kg}$, obtenida al final de los ensayos después del secado en estufa a $103 \pm 2^{\circ} \mathrm{C}$. La densidad básica se calculó por la siguiente formula:

$$
S_{o g}=\frac{M_{0}}{V_{g}}
$$

Para calcular el contenido de humedad se utilizó la siguiente formula:

$$
C H_{g}=\frac{M_{g}-M_{0}}{M_{0}}
$$

donde:

$\mathrm{M}_{\mathrm{g}}=$ masa verde en condición saturada, en $\mathrm{kg}$.

\section{RESULTADOS Y DISCUSION}

En la tabla 3 se muestran los resultados de la variabilidad de la contracción normal transversal del E. nitens. En general la contracción transversal varió entre familias y aumentó de la médula a la corteza de los árboles. 
Entre familias, resultó una menor contracción transversal en la familia 3 y la familia 1 se obtuvo la mayor magnitud de la contracción transversal (Tabla 4), este comportamiento pudo ser debido a las diferencias entre la densidad básica reportada en ambas familias, en la familia 3 con menor contracción transversal, la densidad básica promedio fue de $430 \mathrm{~kg} / \mathrm{m}^{3}$, en cambio en la familia 1 cuya contracción transversal fue mayor, la densidad básica fue de $500 \mathrm{~kg} / \mathrm{m}^{3}$ (Tabla 5).

Tabla 3: Variabilidad radial de la contracción transversal en familias de E. nitens.

\begin{tabular}{|c|c|c|c|c|c|c|}
\hline \multirow{2}{*}{ Familia } & \multirow{2}{*}{ Árbol } & \multirow{2}{*}{ Posición } & \multirow{2}{*}{$\mathrm{CH}(\%)$} & \multicolumn{3}{|c|}{ Contracción normal \% } \\
\hline & & & & Radial & Tangencial & Volumétrica \\
\hline \multirow{9}{*}{1} & \multirow{3}{*}{1} & central & \multirow{9}{*}{14} & & & \\
\hline & & transición & & $3,3(0.25)$ & $4,9(0.29)$ & 8,3 \\
\hline & & lateral & & $4,1(0.25)$ & $6,6(0.27)$ & 10,7 \\
\hline & \multirow{3}{*}{2} & central & & $2,1(0.88)$ & $4,0(1.05)$ & 6,1 \\
\hline & & transición & & $2,5(0.18)$ & $4,5(0.30)$ & 6,9 \\
\hline & & lateral & & $2,6(0.16)$ & $4,9(0.47)$ & 7,5 \\
\hline & \multirow{3}{*}{3} & central & & & & \\
\hline & & transición & & $2,6(0.13)$ & $5,3(0.34)$ & 7,9 \\
\hline & & lateral & & $2,8(0.12)$ & $5,5(0.40)$ & 8,2 \\
\hline \multirow{9}{*}{2} & \multirow{3}{*}{4} & central & \multirow{9}{*}{16} & & & \\
\hline & & transición & & $1,8(0.16)$ & $3,7(0.25)$ & 5,5 \\
\hline & & lateral & & $1,9(0.65)$ & $3,3(0.16)$ & 5,2 \\
\hline & \multirow{3}{*}{5} & central & & $1,0(0.74)$ & $2,6(0.50)$ & 3,6 \\
\hline & & transición & & $1,3(0.36)$ & $4,6(0.26)$ & 5,9 \\
\hline & & lateral & & $1,1(0.26)$ & $3,5(0.94)$ & 4,6 \\
\hline & \multirow{3}{*}{6} & central & & & & \\
\hline & & transición & & 1,5 & 4,4 & 5,9 \\
\hline & & lateral & & $4,1(1.96)$ & $7,7(0.50)$ & 11,8 \\
\hline \multirow{9}{*}{3} & \multirow{3}{*}{7} & central & \multirow{9}{*}{16} & $1,5(0.19)$ & $3,6(0.29)$ & 5,2 \\
\hline & & transición & & $1,9(0.05)$ & $4,9(0.26)$ & 6,8 \\
\hline & & lateral & & $2,7(0.28)$ & $4,8(0.40)$ & 7,6 \\
\hline & \multirow{6}{*}{9} & central & & $1,8(1.06)$ & $4,1(0.28)$ & 5,9 \\
\hline & & transición & & $1,8(0.20)$ & $4,5(0.41)$ & 6,3 \\
\hline & & lateral & & $2,0(1.26)$ & $4,7(1.42)$ & 6,8 \\
\hline & & central & & $1,1(0.33)$ & $2,7(1.03)$ & 3,8 \\
\hline & & transición & & $1,5(0.26)$ & $2,8(0.28)$ & 4,3 \\
\hline & & lateral & & $3,2(0.11)$ & $5,4(0.23)$ & 8,6 \\
\hline \multirow{9}{*}{4} & \multirow{3}{*}{10} & central & \multirow{9}{*}{17} & $1,2(0.36)$ & $3,8(0.26)$ & 5,1 \\
\hline & & transición & & $1,9(0.16)$ & $4,7(0.47)$ & 6,6 \\
\hline & & lateral & & $1,3(0.09)$ & $3,9(0.50)$ & 5,2 \\
\hline & & central & & $2,7(0.35)$ & $5,6(0.77)$ & 8,3 \\
\hline & 11 & transición & & $1,4(0.26)$ & $3,7(0.70)$ & 5,0 \\
\hline & & lateral & & $3,0(0.16)$ & $4,6(1.15)$ & 7,5 \\
\hline & & central & & & & \\
\hline & 12 & transición & & $2,0(0.31)$ & $4,8(0.84)$ & 6,7 \\
\hline & & lateral & & $2,0(0.25)$ & $6,4(1.41)$ & 8,4 \\
\hline & & central & & $2,2(0.16)$ & $3,8(0.22)$ & 6,0 \\
\hline & 13 & transición & & $2,0(0.63)$ & $5,1(0.30)$ & 7,1 \\
\hline & & lateral & & $2,5(0.35)$ & $5,6(0.05)$ & 8,1 \\
\hline & & central & & $1,4(0.02)$ & $4,0(0.35)$ & 5,4 \\
\hline 5 & 14 & transición & 15 & $2,0(0.11)$ & $5,3(0.62)$ & 7,3 \\
\hline & & lateral & & $3,8(0.23)$ & $5,6(2.48)$ & 9,4 \\
\hline & & central & & & & \\
\hline & 15 & transición & & $2,2(0.36)$ & $4,3(0.24)$ & 6,5 \\
\hline & & lateral & & $2,4(0.06)$ & $5,2(0.91)$ & 7,5 \\
\hline
\end{tabular}

()$=$ Desviación standard 
Con la posición radial la contracción tendió a aumentar desde la médula a la corteza. En algunos casos se aprecia una mayor contracción transversal en la zona de transición, por ejemplo en el árbol 5 de la familia 2 y en el árbol 10 de la familia 4. Ambos casos pueden ser debido a una alta contracción tangencial en esta zona, lo que se refuerza por el alto índice de anisotropía de la contracción transversal $(\mathrm{Ctg} / \mathrm{Crd})$ observado en estas zonas, esto es de, 3.7 y 3.1 respectivamente.

Tabla 4: Variabilidad de la contracción, colapso, PIC y Cu entre familias

\begin{tabular}{|c|c|c|c|c|c|c|c|c|c|c|}
\hline Familia & \multicolumn{2}{|c|}{1} & \multicolumn{2}{|c|}{2} & \multicolumn{2}{|c|}{3} & \multicolumn{2}{|c|}{4} & \multicolumn{2}{|c|}{5} \\
\hline Posición & $\mathrm{Rd}$ & $\mathrm{Tg}$ & $\mathrm{Rd}$ & $\mathrm{Tg}$ & $\mathrm{Rd}$ & $\mathrm{Tg}$ & $\mathrm{Rd}$ & $\mathrm{Tg}$ & $\mathrm{Rd}$ & $\mathrm{Tg}$ \\
\hline $\mathrm{CH} \%$ & \multicolumn{2}{|c|}{14} & \multicolumn{2}{|c|}{16} & \multicolumn{2}{|c|}{16} & \multicolumn{2}{|c|}{17} & \multicolumn{2}{|c|}{15} \\
\hline Contracción \% & $\begin{array}{c}2,9 \\
(0.66)\end{array}$ & $\begin{array}{c}5,1 \\
(0.82)\end{array}$ & $\begin{array}{c}1,8 \\
(1.06)\end{array}$ & $\begin{array}{c}4,3 \\
(1.66)\end{array}$ & $\begin{array}{c}1,9 \\
(0.65)\end{array}$ & $\begin{array}{c}4,2 \\
(0.95)\end{array}$ & $\begin{array}{c}1,9 \\
(0.64)\end{array}$ & $\begin{array}{c}4,7 \\
(0.92)\end{array}$ & $\begin{array}{c}2,3 \\
(0.67)\end{array}$ & $\begin{array}{c}4,9 \\
(0.72)\end{array}$ \\
\hline $\begin{array}{c}\text { Contracción } \\
\text { Volumétrica \% }\end{array}$ & \multicolumn{2}{|c|}{8} & \multicolumn{2}{|c|}{6,1} & \multicolumn{2}{|c|}{6,1} & \multicolumn{2}{|c|}{6,6} & \multicolumn{2}{|c|}{7,2} \\
\hline Cn máx & $\begin{array}{c}4,6 \\
(0.98)\end{array}$ & $\begin{array}{c}8,2 \\
(1.09)\end{array}$ & $\begin{array}{c}3,9 \\
(2.11)\end{array}$ & $\begin{array}{c}8,5 \\
(2.37)\end{array}$ & $\begin{array}{c}3,5 \\
(0.99)\end{array}$ & $\begin{array}{c}7,2 \\
(1.32)\end{array}$ & $\begin{array}{c}3,0 \\
(0.89)\end{array}$ & $\begin{array}{c}7,4 \\
(1.35)\end{array}$ & $\begin{array}{c}3,8 \\
(1.06)\end{array}$ & $\begin{array}{c}8,1 \\
(1.17)\end{array}$ \\
\hline Colapso & $\begin{array}{c}2,0 \\
(1.11)\end{array}$ & $\begin{array}{c}3,6 \\
(1.40)\end{array}$ & $\begin{array}{c}2,9 \\
(1.48)\end{array}$ & $\begin{array}{c}6,3 \\
(3.67)\end{array}$ & $\begin{array}{c}2,0 \\
(0.86)\end{array}$ & $\begin{array}{c}3,6 \\
(1.10)\end{array}$ & $\begin{array}{c}2,5 \\
(0.94)\end{array}$ & $\begin{array}{c}3,4 \\
(0.97)\end{array}$ & $\begin{array}{c}2,7 \\
(1.04)\end{array}$ & $\begin{array}{c}5,0 \\
(2.00)\end{array}$ \\
\hline $\begin{array}{c}\text { Colapso } \\
\text { volumétrico \% }\end{array}$ & \multicolumn{2}{|c|}{5,1} & \multicolumn{2}{|c|}{9,2} & \multicolumn{2}{|c|}{5,6} & \multicolumn{2}{|c|}{5,9} & \multicolumn{2}{|c|}{7,7} \\
\hline PIC & $\begin{array}{c}28,1 \\
(0.40)\end{array}$ & $\begin{array}{c}28,6 \\
(0.95)\end{array}$ & $\begin{array}{c}33,5 \\
(0.35)\end{array}$ & $\begin{array}{c}33,6 \\
(0.18)\end{array}$ & $\begin{array}{c}32,4 \\
(0.45)\end{array}$ & $\begin{array}{c}31,4 \\
(0.34)\end{array}$ & $\begin{array}{c}32,7 \\
(0.55)\end{array}$ & $\begin{array}{c}34,0 \\
(0.41)\end{array}$ & $\begin{array}{c}29,8 \\
(0.44)\end{array}$ & $\begin{array}{c}29,9 \\
(0.22)\end{array}$ \\
\hline $\mathrm{Cu}$ & $\begin{array}{c}0,12 \\
(0.03)\end{array}$ & $\begin{array}{c}0,21 \\
(0.03)\end{array}$ & $\begin{array}{c}0,13 \\
(0.07)\end{array}$ & $\begin{array}{c}0,26 \\
(0.05)\end{array}$ & $\begin{array}{c}0,10 \\
(0.03)\end{array}$ & $\begin{array}{c}0,20 \\
(0.03)\end{array}$ & $\begin{array}{c}0,07 \\
(0.02)\end{array}$ & $\begin{array}{c}0,16 \\
(0.03)\end{array}$ & $\begin{array}{c}0,10 \\
(0.03)\end{array}$ & $\begin{array}{c}0,22 \\
(0.04)\end{array}$ \\
\hline
\end{tabular}

( )=Desviación standard

Se observa además en la Tabla 3 que la familia 2 presenta menor contracción radial, con un promedio de $1.8 \%$, para los tres árboles y las tres secciones (central, transición y lateral), siendo el árbol 5 en la sección central la de menor magnitud en la contracción radial, esto es, $1.0 \%$. Mientras que la familia 1 muestra mayor contracción radial, obteniendo un promedio de $2.9 \%$, siendo en el árbol 1 , en la posición lateral, donde se obtuvo la mayor magnitud de la contracción radial, esto es, $4.1 \%$. 
Tabla 5: Densidad básica de las familias de E. nitens

\begin{tabular}{|c|c|c|c|c|c|c|}
\hline \multirow{2}{*}{ Familia } & \multirow{2}{*}{ Árbol } & \multicolumn{5}{|c|}{ S o,g $\left(\mathrm{kg} / \mathrm{m}^{3}\right)$} \\
\hline & & Central & Transición & Lateral & Árbol & Familia \\
\hline \multirow{3}{*}{1} & 1 & & $\begin{array}{l}480 \\
(10)\end{array}$ & $\begin{array}{l}510 \\
(10)\end{array}$ & 500 & \multirow{3}{*}{500} \\
\hline & 2 & $\begin{array}{l}460 \\
(20)\end{array}$ & $\begin{array}{l}450 \\
(10)\end{array}$ & $\begin{array}{l}530 \\
(10)\end{array}$ & 480 & \\
\hline & 3 & & $\begin{array}{l}480 \\
(10)\end{array}$ & $\begin{array}{l}540 \\
(10)\end{array}$ & 510 & \\
\hline \multirow{3}{*}{2} & 4 & & $\begin{array}{l}390 \\
(20)\end{array}$ & $\begin{array}{l}400 \\
(10)\end{array}$ & 400 & \multirow{3}{*}{450} \\
\hline & 5 & $\begin{array}{l}400 \\
(40)\end{array}$ & $\begin{array}{l}390 \\
(30)\end{array}$ & $\begin{array}{l}420 \\
(40)\end{array}$ & 400 & \\
\hline & 6 & & $\begin{array}{c}500 \\
(10)\end{array}$ & $\begin{array}{l}580 \\
(20)\end{array}$ & 540 & \\
\hline \multirow{3}{*}{3} & 7 & $\begin{array}{l}430 \\
(30)\end{array}$ & $\begin{array}{l}420 \\
(20)\end{array}$ & $\begin{array}{l}450 \\
(10)\end{array}$ & 430 & \multirow{3}{*}{430} \\
\hline & 8 & $\begin{array}{l}420 \\
(10)\end{array}$ & $\begin{array}{l}400 \\
(20)\end{array}$ & $\begin{array}{l}500 \\
(10)\end{array}$ & 440 & \\
\hline & 9 & $\begin{array}{l}400 \\
(20)\end{array}$ & $\begin{array}{l}380 \\
(10)\end{array}$ & $\begin{array}{l}480 \\
(10)\end{array}$ & 420 & \\
\hline \multirow{3}{*}{4} & 10 & $\begin{array}{l}400 \\
(10)\end{array}$ & $\begin{array}{l}390 \\
(10)\end{array}$ & $\begin{array}{l}430 \\
(30)\end{array}$ & 410 & \multirow{3}{*}{440} \\
\hline & 11 & $\begin{array}{l}490 \\
(10)\end{array}$ & $\begin{array}{l}390 \\
(30)\end{array}$ & $\begin{array}{l}410 \\
(20)\end{array}$ & 430 & \\
\hline & 12 & & $\begin{array}{l}420 \\
(30)\end{array}$ & $\begin{array}{l}510 \\
(20)\end{array}$ & 470 & \\
\hline \multirow{3}{*}{5} & 13 & $\begin{array}{l}420 \\
(10)\end{array}$ & $\begin{array}{l}420 \\
(20)\end{array}$ & $\begin{array}{l}480 \\
(20)\end{array}$ & 440 & \multirow{3}{*}{440} \\
\hline & 14 & $\begin{array}{l}420 \\
(20)\end{array}$ & $\begin{array}{l}400 \\
(20)\end{array}$ & $\begin{array}{l}480 \\
(20)\end{array}$ & 430 & \\
\hline & 15 & & $\begin{array}{l}410 \\
(10)\end{array}$ & $\begin{array}{l}480 \\
(20)\end{array}$ & 450 & \\
\hline
\end{tabular}

$($ )=Desviación standard

Con respecto a la contracción tangencial, la familia 3 es la que presenta la menor contracción $3.1 \%$, mientras que en el árbol 9 se observa la menor magnitud de contracción tangencial, con un valor de $2.7 \%$. La familia 1 es la que en promedio presenta la mayor magnitud de contracción tangencial, siendo en el árbol 1, en la posición lateral, donde se alcanza una contracción tangencial de $6.6 \%$. 
La contracción normal máxima fue en promedio para todas las familias de 3.8\% y $7.9 \%$ en dirección radial y tangencial respectivamente, con un índice de anisotropía de la contracción transversal $\mathrm{Ctg} / \mathrm{Crd}=2.08$, estos valores son un poco más bajos que los reportados por Sepúlveda y Urrutia (1996), lo que es consistente con una pequeña menor densidad básica reportada en estos ensayos. En estos ensayos la densidad básica promedio fue de $450 \mathrm{~kg} / \mathrm{m}^{3}$ (Tabla 5) en comparación a $460 \mathrm{~kg} / \mathrm{m}^{3}$ reportado por Sepúlveda y Urrutia (1996).

\section{Variabilidad del colapso}

La variabilidad del colapso se muestra en la Tabla 6. Se observa heterogeneidad en la magnitud del colapso entre las familias y con la posición radial.

Entre familias el colapso volumétrico varia entre 5.1 y $9.2 \%$ entre las familias 1 y 2 respectivamente. Es notable en todo caso la baja magnitud del colapso en la familia 1 cuyo contenido de humedad es de $14 \%$, siendo el menor entre todas las familias (Tabla 4). En el caso de la familia 2 el mayor colapso es influenciado por el alto colapso observado particularmente en el árbol 5, en este caso, el colapso volumétrico varió entre 7 y $16.7 \%$, con un contenido de humedad de $16 \%$.

Con la posición radial el colapso tendió a aumentar desde médula a corteza, esta tendencia del colapso con la posición radial ha sido reportada en la literatura (Chafe et al. 1992). En la familia 2 los árboles 4 y 5 mostraron un aumento del colapso desde la zona central a la de transición y luego una disminución en la zona lateral, igual comportamiento presento el árbol 10 de la familia 4; este comportamiento es similar al reportado para la contracción en los árboles 4 y 10 . La mayor intensidad del colapso en la zona de transición en estos árboles se debe a la menor densidad básica reportada en esta zona para los árboles 4, 5 y 10 (Tabla 5). En cambio el árbol 11 de la familia 4 presento el colapso más intenso en la zona central y un menor colapso en la zona de transición, en este caso, la disminución del colapso en la zona de transición puede ser debida a una mayor densidad normal reportada en esta zona del árbol, esto es, $700 \mathrm{~kg} / \mathrm{m}^{3}$, en comparación a $620 \mathrm{~kg} / \mathrm{m}^{3}$ y $650 \mathrm{~kg} / \mathrm{m}^{3}$ reportadas en la zona central y lateral respectivamente (Leandro et al. 2008b).

Los valores de colapso radial, en distintas posiciones indican que existe menor intensidad de colapso en la familia 3, presentando en promedio un 2.0\%, siendo el árbol 9 el que presentó el menor colapso, con un total de $1.2 \%$ en su sección central. Mientras que en la familia 2 se observa mucho más intenso el colapso con un promedio de $2.9 \%$, alcanzando en el árbol 6 hasta un 5.4\% de colapso en la sección lateral.

Se observa que el menor colapso en la dirección tangencial se presenta en la familia 1, entregando un total de 3.6\% como promedio, obteniendo en la sección de transición del árbol 3 solo un $1.6 \%$ de colapso. El colapso más intenso se encuentra en la familia 2, con un total de $6.3 \%$ de colapso tangencial, alcanzando en el árbol 5, un $12.4 \%$ de colapso en la sección de transición. 
Tabla 6: Variabilidad radial del colapso transversal en las familias de E. nitens.

\begin{tabular}{|c|c|c|c|c|c|c|}
\hline \multirow{2}{*}{ Familia } & \multirow{2}{*}{ Árbol } & \multirow{2}{*}{ Posición } & \multirow{2}{*}{$\mathrm{CH}(\%)$} & \multicolumn{3}{|c|}{ COLAPSO \% } \\
\hline & & & & Radial & Tangencial & Volumétrico \\
\hline \multirow{9}{*}{1} & \multirow{3}{*}{1} & central & \multirow{9}{*}{14} & & & \\
\hline & & transición & & $3,2(0.36)$ & $3,2(0.66)$ & 6,4 \\
\hline & & lateral & & $3,8(0.36)$ & $6,1(0.74)$ & 9,9 \\
\hline & \multirow{3}{*}{2} & central & & $1,7(1.36)$ & $2,5(0.80)$ & 4,2 \\
\hline & & transición & & $1,3(0.04)$ & $2,8(0.13)$ & 4,1 \\
\hline & & lateral & & $1,6(0.29)$ & $2,8(0.35)$ & 4,4 \\
\hline & \multirow{3}{*}{3} & central & & & & \\
\hline & & transición & & $0,7(0.16)$ & $1,6(0.21)$ & 2,3 \\
\hline & & lateral & & $1,4(0.11)$ & $3,1(0.28)$ & 4,5 \\
\hline \multirow{9}{*}{2} & \multirow{3}{*}{4} & central & \multirow{9}{*}{16} & & & \\
\hline & & transición & & $1,6(0.30)$ & $4,6(0.97)$ & 6,2 \\
\hline & & lateral & & $1,8(0.98)$ & $2,7(0.29)$ & 4,5 \\
\hline & \multirow{3}{*}{5} & central & & $2,1(0.20)$ & $4,9(0.56)$ & 7,0 \\
\hline & & transición & & $4,3(0.71)$ & $12,4(3.65)$ & 16,7 \\
\hline & & lateral & & $3,1(0.83)$ & $8,2(1.64)$ & 11,3 \\
\hline & \multirow{3}{*}{6} & central & & & & \\
\hline & & transición & & $1,7 *$ & $2,4^{*}$ & 4,1 \\
\hline & & lateral & & $5,4(1.25)$ & $8,9(1.17)$ & 14,3 \\
\hline \multirow{9}{*}{3} & \multirow{3}{*}{7} & central & \multirow{9}{*}{16} & $1,1(0.09)$ & $2,4(0.50)$ & 3,6 \\
\hline & & transición & & $2,4(0.18)$ & $3,2(0.69)$ & 5,6 \\
\hline & & lateral & & $3,6(0.77)$ & $5,2(0.48)$ & 8,9 \\
\hline & \multirow{3}{*}{8} & central & & $1,3(0.67)$ & $2,9(0.12)$ & 4,1 \\
\hline & & transición & & $1,4(0.17)$ & $3,5(0.61)$ & 4,9 \\
\hline & & lateral & & $2,6(1.65)$ & $5,7(3.05)$ & 8,2 \\
\hline & \multirow{3}{*}{9} & central & & $1,2(0.30)$ & $3,0(0.17)$ & 4,2 \\
\hline & & transición & & $1,6(0.16)$ & $3,0(0.33)$ & 4,6 \\
\hline & & lateral & & $2,5(0.33)$ & $3,5(0.16)$ & 5,9 \\
\hline \multirow{9}{*}{4} & \multirow{3}{*}{10} & central & & $1,4(0.22)$ & $2,9(0.31)$ & 4,2 \\
\hline & & transición & & $3,2(0.79)$ & $3,0(0.93)$ & 6,2 \\
\hline & & lateral & & $1,3(0.79)$ & $2,3(0.43)$ & 3,6 \\
\hline & & central & & $3,8(0.80)$ & $3,5(2.89)$ & 7,4 \\
\hline & 11 & transición & 17 & $1,7(2.03)$ & $3,6(1.58)$ & 5,3 \\
\hline & & lateral & & $3,3(0.51)$ & $2,5(1.89)$ & 5,8 \\
\hline & & central & & & & \\
\hline & 12 & transición & & $2,9(1.00)$ & $4,3(1.72)$ & 7,2 \\
\hline & & lateral & & $2,4(0.26)$ & $5,2(1.89)$ & 7,6 \\
\hline & & central & & $2,0(0.54)$ & $3,7(0.11)$ & 5,7 \\
\hline & 13 & transición & & $2,4(0.35)$ & $5,2(0.57)$ & 7,6 \\
\hline & & lateral & & $4,0(1.50)$ & $7,5(0.20)$ & 11,5 \\
\hline & & central & & $0,9(0.12)$ & $2,0(0.16)$ & 2,9 \\
\hline 5 & 14 & transición & 15 & $2,6(0.22)$ & $3,4(0.91)$ & 6,0 \\
\hline & & lateral & & $3,9(1.06)$ & $7,9(4.69)$ & 11,8 \\
\hline & & central & & & & \\
\hline & 15 & transición & & $2,8(0.40)$ & $4,6(0.71)$ & 7,4 \\
\hline & & lateral & & $3,3(1.19)$ & $5,5(0.51)$ & 8,8 \\
\hline
\end{tabular}

\section{( )=Desviación Standard}

* =el árbol 6 en la sección de transición presenta solo una probeta, por lo que no se le calculo la desviación standard 


\section{Variación del punto de intersección de la contracción (PIC) y coeficiente de contracción unitario (Cu)}

Los resultados del PIC y del Cu se muestran en la tabla 4. Se observa que el PIC y el Cu varían entre las familias. Las familias 2, 3 y 4 presentan los PIC más altos. En particular el PIC en la dirección tangencial de la familia 4 es de 34\%. Entre las familias 1 y 5 el menor PIC lo presenta la familia 1 en la dirección radial esto es, $28.1 \%$. El Cu en cambio varía entre 0.07 en la dirección radial de la familia 4 y 0.26 en la dirección tangencial de la familia 2. Esto implica que la familia 4 es la más estable dimensionalmente.

Se aprecia que el PIC y el $\mathrm{Cu}$ son de mayor magnitud en la dirección tangencial excepto el PIC en la familia 3. Este comportamiento es debido a la anisotropía de la contracción transversal. La magnitud del PIC y del Cu resultó un poco más baja que los reportados por Sepúlveda y Urrutia (1996), debida a la mayor edad y probablemente a un mayor grado de duraminización de la madera en estos ensayos.

\section{CONCLUSIONES}

La contracción y el colapso en Eucalyptus nitens tienden a aumentar con la posición radial, entre árboles y entre familias. Una alta contracción e intenso colapso son observados en la zona central y lateral en asociación con una baja densidad en esta zonas.

De acuerdo a esto resultados, el mejor comportamiento en contracción y colapso es presentado por la familia 1, en particular los árboles 2 y 3 muestran baja contracción y colapso. Este comportamiento es favorecido por una relativamente alta densidad básica.

\section{AGRADECIMIENTOS}

Los autores agradecen a la empresa CMPC División Forestal por la donación de la madera necesaria para estos ensayos experimentales.

\section{BIBLIOGRAFIA}

Chafe, S.; Barnacle, J.; Hunter, A.; Ilic, J.; Northway, R.; Rozsa, A. 1992. Collapse: An introduction. CSIRO, Division of Forest Products. 9pp.

Hamilton, M.; Raymond, C.; Harwood, C.; Potts, B. 2009. Genetics variation in Eucalyptus nitens pulpwood and wood shrinkage traits. Tree Genetics \& Genomes 5:307-316.

INFOR. 1989. Eucalyptus. Principios de silvicultura y manejo. INFOR, Santiago, Chile

INFOR. 2004. Eucalyptus nitens en Chile: Procesos industriales de la madera. Informe Técnico No 164. INFOR, Santiago, Chile. 
INN. 1984. Determinación de la contracción de la madera. Norma chilena oficial 176/3 of 84. Instituto Nacional de Normalización, Chile.

INN. 1988. Determinación de la densidad de la madera. Norma chilena oficial 176/2 of 86. Instituto Nacional de Normalización, Chile.

Leandro, L.; Ananías, R.; Cloutier, A. ; Díaz-Vaz, J.E.; Bermedo, M.; Sanhueza, R.; Lasserre.; J.P. 2008a. Estudio preliminar de las grietas internas dentro de los anillos de madera inicial y su relación con algunas características de la estructura anatómica y la densidad en Eucalyptus nitens. Interciencia 33(11): 829-834.

Leandro, L.; Ananías, R.A.; Cloutier, A. 2008b. Correlaciones entre propiedades anatómicas y densidad normal en Eucalyptus nitens. Paper presentado en el IV Congreso Chileno de Ciencias Forestales, Fac. Cs. Forestales, Universidad de Talca, Chile. Octubre 2008. 18 pp.

Leandro, L.; Cloutier, A.; Ananías, R.A. 2007. Propiedades de familias de Eucalyptus nitens relacionadas con el secado. Algunos resultados iniciales. Poster presentado en el día del CRB. Université Laval, Canadá.

Rozas, C.; Sánchez, R.; Pineda, P. 2005. Secado de Eucalyptus nitens y globulus y su aprovechamiento en blocks, pisos y muebles. Maderas. Ciencia y tecnología 7(2)109-120.

Sepúlveda, P.; Urrutia, W. 1996. Ensayos de contracción y medición de la velocidad de propagación del ultrasonido en la madera de Eucalyptus nitens. Seminario titulación. Dpto. Ingeniería en Maderas. Fac. de Ingeniería. Universidad del Bío-Bío. Concepción. Chile

Washusen, R.; Innes, T. 2008. Processing plantation Eucalypts for high-value timber. In plantation Eucalypts for high-value timber. Ed. A.G. Brown and C.L. Beadle. RIRDC Publication No8: 92-118.

Washusen, R.; Harwood, C.; Morrow, A.; Valencia, J.C.; Volker, P., Wood, M.; Innes, T.; Ngo, D.; Northway, R., Bojadzic, M. 2008. Eucalyptus nitens thinning trial: Solid wood quality and processing performance using conventional processing strategies. Forest and Wood Products Australia. Project number PN07.3019. 